\title{
Nurse Home Visit Program to Promote Parenting In Brazil: Premises And Planning
}

luciola siqueira ( $\square$ luciola.demery@gmail.com )

Universidade de Sao Paulo https://orcid.org/0000-0001-5087-9824

\section{Anna Maria Chiesa}

Universidade de Sao Paulo

\section{Euripedes Miguel}

Universidade de Sao Paulo

\section{Leticia Silva}

Universidade de Sao Paulo

Sharonya Vadakattu

University of Pennsylvania

Fernanda Alarcao

Universidade de Sao Paulo

Lislaine Fracolli

Universidade de Sao Paulo

\section{Research article}

Keywords: Home visit, Pregnancy in adolescence, Parenting, Early childhood, Nursing

Posted Date: June 17th, 2020

DOI: https://doi.org/10.21203/rs.3.rs-34304/v1

License: (c) (i) This work is licensed under a Creative Commons Attribution 4.0 International License.

Read Full License 


\section{Abstract}

\section{Background}

Promoting good parenting has been a challenge for public policies since child development became part of the Sustainable Development Goals until 2030. Home visitation programs have been proved to be effective in supporting the most vulnerable families, especially the vulnerable, first time pregnant's teenagers, in acquiring skills and incorporating parenting practices that promote integral child development. The Young Mothers Caregivers is an innovative, experimental program, implemented in the context of a RCT. This paper aims to analyze the fidelity of the HVs, regarding the planning and the operative framework of the young Mothers Cargivers' protocol.

\section{Methods}

This qualitative study was conducted by content analysis from nurse's records of $390 \mathrm{HV}$ selivered for 39 teenagers, attended by YMCP. The software Wordart was used to visualize the frequency of themes. The analysis examined, 1) which priority areas were most focused on in HV records, and 2) which themes were prominent in each content priority area, according to the five axes that compose the bases of the interventions.

\section{Results}

The first trimester of HV focused on "Care with Health" axis (specially "Nutrition"); along with "Life Course" axis, "Familiar\&Social Network", "Environment" and "Parenting". In the second trimester of HV, again "Care with Health" axis (primarily focused on "Nutrition" and "Birth") was identified in records, as was the concept of "Parenting" ("Praise", "Attachment", and "Expressing Feelings"). In the third trimester of HV, "Care with Health" axis (primarily through Breastfeeding and Birth) and "Parenting" axis(through Praise, Attachment, and Readiness to Address Concerns) were prominent.

\section{Conclusions}

The content priorities raised in each HV trimester, with different themes within each category, indicating the capacity to adjust the protocol according to the youth interest. At different times, some categories were more highlighted than others. The study found recurring emphasis on praise and the nurse's ability to address concerns, underscoring the importance of a strong relationship among the nurse visitor and the young mother.

\section{Relevance to Practice}

Data highlights the role of responsive communication and participant's centered needs as skills for nurses that delivers HV. This is a crucial aspect to structure the monitoring and supervision axes of the Program, for further scaling up. 


\section{Background}

Home Visitation programs, with a focus in the improvement of parenting skills, positively influence childhood development and family functioning by enabling relationships that are more responsive and sensitive between parents and children. ${ }^{(1,2)}$

Many different home visitation programs around the world which fortify parenting competencies have been proven to be effective, for some relevant outcomes in early childhood, including: maternal health; child health; positive parenting practices, child development and school performance; reduction of child abuse; economic self-sufficiency of the family; recognise and use the social services present in the neighborhood; reduction in juvenile delinquency, family violence and crime ${ }^{(3)}$. There is still a remaining question about the specific contribution of those programs to the families living in low-and - middleincome countries. ${ }^{(4)}$

The implementation of Home visitation programs with a focus on parenting in developing countries, like Brazil, is recent. Thus, besides the difficulties related to the availability of resources to implement these programs, socioeconomic vulnerability of the population creates a complex web of factors that impact child development. ${ }^{(5)}$

Although programs that aim to promote parenting competencies are structured in different forms (concerning frequency of intervention, content of visits, professional training), the home visit (HV) approach is one of the most common forms of offering this kind of intervention ${ }^{(6)}$. HV is a good choice for programs with a focus on parenting, considering that the effects of parental competencies are seen within families and households ${ }^{(7)}$. Once the professional is inside the residence, is better to observe up close the socioeconomic reality, see the stimuli offered to the children in the environment, and note the comprehension of health needs ${ }^{(9)}$. Facing the objectives to building parenting competencies, home visitation programs consider registered nurses as competent professionals for deliver the intervention. Such professionals forge lasting relationships with the participants and create a foundation of trust ${ }^{(9)}$, beyond promoting emotional well-being and self-sufficiency of the family. ${ }^{(10)}$

The dynamic and contextual nature of $\mathrm{HV}$ requires that they be organized based on the balance between the content that needs to be delivered and the flexibility that the demands of the home bring. Therefore, knowing the topics discussed during HV and assessing their fidelity to the objectives of the intervention program are essential to ensure the achievement of results. ${ }^{(11,12)}$.

When certain themes, that were not initially part of the Home Visitation program's intention, emerge The focus frequently comes like the range of the themes which are not "planned and predicted" by the program's protocol. ${ }^{(13)}$ Given this, adopting strategies to monitor HVs allows to identify the RN's capacity of effectively work with different themes regarding both the individual and the families receiving the intervention. ${ }^{(14)}$ 
The objective of this study is to describe the content and priorities of prenatal HVs conducted by 3 nurses as part of the analysis of fidelity of Young Mothers Caregivers Program (YMCP).

\section{Methods}

This study on the implementation of a pilot of a home visit program focused on adolescent mothers, analyzed the notes contained in the visit protocol filled out by visiting nurses hired to work in the Young Mothers Caregivers Program. (YMCP). This qualitative and longitudinal study was conducted by the coordinator team of the YMCP as well as pos graduated students, all female researchers from the university and trained in qualitative analysis before. The research team was informed by the YMCP participating in research meetings promoted by the coordinators. The analysis focused documents collected from the Home Visits registers filled by the nurses during (month, years, from 35 participants) and content analysis techniques to examine the frequency and prevalence of words and themes throughout the nurse's notes of prenatal visits of the program.

The protocols, which address the adolescents assisted by YMCP, used as the source for data collection in this study. These protocols contained the records of $390 \mathrm{HVs}$, representing the total number of visits performed during the prenatal period of 35 participants who remained in the program.

The protocols' records were organized and entered into spreadsheets using the software Excel 2013. From these worksheets, specifically the study examined the records referring to the actions that took place during each visit and the actions planned for future visits. These records of actions were analyzed based on the frequency with which they appeared.

The study used content analysis, looking specifically at recurring themes and using word clouds as a technique, as an analytical strategy to describe and interpret the content of the documents and text analyzed. This assisted with the interpretation of messages and brought a new level of understanding to the data's meaning that goes beyond the literature. The literature describes the process of analyzing content in five steps: 1) Preparing the information; 2) "Unitization" or transforming content into units; 3 ) Categorizing or classifying the units into categories; 4) Description; 5) Interpretation (15).

In this study, the HV records were organized in Excel spreadsheets. Followed by material exploration and categorization into themes, and then processing and interpretation of results. The analysis of the contents of the reports were followed by: first reading of each report and then were elaborated themes according to the main subject addressed in that action. Each single home visit can generate more than one theme if the nurse describes more than one action to be developed. The themes were extracted during sequential readings of the material, not previously established. The study analyzed the actions that were both planned and executed during the three trimesters of pregnancy, which classified as the following: 1 st -6 th HV as the first trimester; 7 th to 11 th HV as the second trimester; and 12th -17 th HV as the third trimester. 
The creation of word clouds utilized as a complement to the thematic analysis. This approach allowed for the visualization of words extracted from the text in a graphic form. In these diagrams, the words with a higher frequency of appearances in the text appear in larger fonts than the other words, thus creating an image of words of various sizes. Word clouds are a useful tool for quantitatively analyzing spoken, written and / or transcribed texts. ${ }^{(16)}$ In this study, wordart.com, one of the free tools for word cloud creation, used with the following configurations: organization by common English terms and use of a layout with a color scheme.

The utilization of these visualization techniques for qualitative data requires attention to ensure that the qualitative information is not simply reduced and simplified to counts of frequency. Thus, it is recommended to include excerpts from the analyzed content to not lose the essence and depth which characterize qualitative research. ${ }^{(16)}$

\section{Description}

YMCP was part of the pilot stage of the research project, "The Effect of a Home-visitation Program for Young Mothers Regarding Childhood Development", a randomized control clinical trial, which began in August of 2015 and ended in May of 2018, as part of an inter-institutional partnership and financed in part by development agencies (http://inpd.org.br/?page_id=4062). The study was registered on clinicaltrial.gov with the code NCT02807818 and depended on up to three nurses for its implementation.

YMCP was a pilot initiative using HVs for adolescents between the ages of 14 and 19 who live in a socioeconomically vulnerable situation in the West Zone of the municipality of São Paulo, Brazil. The program's structured axes to promote better development in early childhood are: improving health outcomes; promoting sensitive and responsive relationships (positive parenting); improve domestic environment; strengthen familiar and social net; and improving the individual capabilities to achieve a better integration of the labor market into life. YMCP offered to 40 adolescent first-time mothers of a low socioeconomic level, from the first trimester of pregnancy up until the child's second birthday.

The RN received initial training, weekly supervision, and special training in filling out the protocol for the systematization of actions, which especially constructed for the program ${ }^{(17)}$.

YMCP treated the following axes as guidelines for its approach to young mothers and their family ${ }^{(18)}$ :

1. Health and care: support and stimulate maternal actions aimed at maintaining and improving maternal and child health;

2. Environmental health: help the teenage mother to identify, organize, and use the physical and relational resources that could help her in the care of the child, taking into consideration the different necessities of the development process;

3. Parenting and bond: to help the teenage mother and father of the baby develop knowledge and capabilities to support with confidence the comprehensive health and development of the child; 
4. Family and social support: help the teenage mother to understand and manage her relationships with people who are beneficial to the mother and the needs of the child

5. Life course: to help the teenage mother identify relevant goals for her life to increase the opportunities for social and economic independence.

The total frequency of visits planned during pregnancy was up to 17, depending on the date of entry of the mother into the program and/ or her availability to accommodate the visitor.

\section{Results}

Reports from $390 \mathrm{HVs}$ delivered for 35 adolescents were analyzed. There were $180 \mathrm{HVs}$ in the first trimester of pregnancy (1st - 6th HV), $133 \mathrm{HVs}$ in the second trimester of pregnancy (7th to 11th HV); and $77 \mathrm{HVs}$ in the third trimester of pregnancy (12th - 17th HV). There is a clear reduction in the number of visits, which is due to factors such as abandoning the study or lack of availability of the mother. Table 1 presents extracts from the nurses' reports, the number of apparitions and their corresponding categorization. In this table, the sixteen most relevant themes in terms of frequency discussed. 
Table 1

Categorization, frequency of themes and reports of visiting nurses. Sao Paulo, 2020.

\begin{tabular}{|c|c|c|}
\hline Themes & $\mathbf{n}$ & Reports of visiting nurses \\
\hline Nutrition & 86 & $\begin{array}{l}\text { "Reinforce on the importance of keeping up with weight and healthy eating." } \\
\text { "Perform food recall and guide healthy eating, weight gain and body mass } \\
\text { index (BMI)." "Build daily food plan on a table for better viewing." }\end{array}$ \\
\hline Praise & 64 & $\begin{array}{l}\text { "Praise health care." "Praise decrease in cigarette use and recommend } \\
\text { cessation." "Praise attachment and behavior change." "Strengthen and praise } \\
\text { the ability to communicate." }\end{array}$ \\
\hline Feelings & 46 & $\begin{array}{l}\text { "Stimulating expression of feelings towards his mother and the care that was } \\
\text { offered to him in childhood." "Use dramatization so she can work out better } \\
\text { ideas and feelings." "Feeling of solitude to be worked on." "Encourage the } \\
\text { pregnant woman to express her feelings regarding the separation of the } \\
\text { parents and the absence of the mother in childhood." }\end{array}$ \\
\hline
\end{tabular}

Attachment $\quad 45$ "Encourage attachment through communication and caring in the belly." "Improving attachment between pregnant and fetus." "Work attachment using the last performed imaging exam." "Stimulate the current attachment pattern." "Strengthen attachment signs by praising and speaking of importance."

Health Care $\quad 44 \quad$ "Investigate prenatal progress and linkage at the health center." "Encourage going to health check for appointment scheduling." "Strengthen self-care skills with health care guidelines." "Guide folic acid use strategy."

Delivery 43 "Investigate the research of types of labor to begin the construction of the birth plan." "Strengthen action plan for childbirth." "Provide indication of teaching material about childbirth and testimony of young mothers." "It assists in the organization of the maternity suitcase." "Be available for doubt about delivery."

Life course $\quad 40 \quad$ "Strengthen short-term living plans." "Work life project and independence of parents." "Stimulate search for alternatives to financial independence."

"Explore your intentions and expectations about resuming studies." "Propose written planning of steps to enroll in distance learning."

Social

Network

40 "Make genogram and ecomap to talk about social network." "Guide on triggering social network for support with food." "Talk to the pregnant woman using the map of the region's resources and linking to their needs."

Breastfeeding $27 \quad$ "Start breastfeeding talk." "Continue encouraging breastfeeding." "Work out the positions for breastfeeding and get correct using doll."

Tobacco 20 "Propose harm reduction with tobacco and marijuana use." "Guidance on decreasing tobacco use." "Guiding on the risks of using rope cigarette."

Environment $\quad 19$ "Address household hygiene issues and division of household chores." "Check progress of the preparation of the place where the baby will be." "Offering media about gestation and how to prepare the environment to receive the baby." "Talk about safe and healthy environment for baby development."

Relationship 19 "Work relationship with grandmother." "Clarify relationship with mother." "Investigate relationship history with baby's father." "Investigate history of previous relationship as possible cause of attachment difficulty." 


\begin{tabular}{|c|c|c|}
\hline Themes & $\mathbf{n}$ & Reports of visiting nurses \\
\hline Family & 17 & $\begin{array}{l}\text { "Talk to her mother about communication during the visit." "Not feeling } \\
\text { comfortable with her mother close at the time of the visit, but she does not } \\
\text { have to ask to leave. Try the next one another place to talk. " }\end{array}$ \\
\hline Empowerment & 14 & $\begin{array}{l}\text { "Strengthen her ability to position herself before her husband, clarifying that } \\
\text { her body has its own rhythm and that it concerns her." "Stimulate the } \\
\text { expression of your intentions and expectations of being more independent." }\end{array}$ \\
\hline Doubt & 14 & $\begin{array}{l}\text { "Be available by phone and messages." "Make room for the pregnant woman } \\
\text { to bring her doubts." "Be available to clarify doubts." }\end{array}$ \\
\hline Paternity & 8 & $\begin{array}{l}\text { "Encourage dialogue with the baby's father." "Hold conversations regarding } \\
\text { your relationship with the baby's father." "Guidance on the importance of the } \\
\text { father of the child in the follow-up of pregnancy." "Investigate and encourage } \\
\text { partner participation in prenatal routines." }\end{array}$ \\
\hline
\end{tabular}

Table 1

For the formation of the word clouds, themes grouped by the gestational trimester corresponding to each participant at the time of the HV in question.

\section{First Trimester}

This phase includes the 1st - 6 th HV, over the first three months of the adolescent's participation in the program. At this point, one can see the efforts on the part of the nurse to plan and carry out actions related to the premises of the program. We highlight here a focus on nutrition, life course, and social network. Some actions also relate to the expression of feelings of acceptance of the pregnancy and the development of attachment. At the end of this phase, attitudes of positive reinforcement were observed, with praise directed at behavioral changes - primarily related to reductions in smoking, interpersonal relationships, and self-care.

\section{FIGURE 1}

\section{Second Trimester}

During this phase, which covers the 7th $-11 \mathrm{th} \mathrm{HV}$, the nurse elaborates upon themes that introduced earlier on. Nutrition again highlighted as the largest theme concerning the planning of actions, followed by childbirth, and praise. Themes related to feelings (including stress, affection, attachment, family, and relationship with significant others) were all approached in detail, primarily because there is by this point a more developed bond with the nurse. Thus, the participant feels more willing to express her feelings.

FIGURE 2

\section{Third Trimester}


The themes that emerged from the visits preceding the due date focused on preparation for birth. The visitor made themselves available to address concerns and doubts, and breastfeeding was a frequent topic, along with other practical activities. This period also was an opportunity to strengthen the bond between the mother and the baby, through encouraging attachment and the expression of feelings.

FIGURE 3

\section{Discussion}

The findings show that the themes most prevalent in the planning for future visits related with the program's premises, highlighting the bond among the nurse and the adolescent mother in the program.

The axis "Health care" reflected in actions related to nutrition, expression of feelings, consumption of tobacco, and care with physical health. An emphasis can be seen on actions related to nutrition, primarily regarding weight control and the importance of a healthy diet. This can be understood related to the social vulnerability of the sample that was at risk for malnutrition even previously of the pregnancy. The YMP was based on the assumption that health data could be assessed by the Pregnant Health Booklet filled by health professionals in the public services, proposed at national level. Nevertheless, despite the vulnerability of our population, the proposed schedule was not followed for every pregnant youth. This circumstance created a problem for the nurse visitor to evaluate the physical dimension of pregnancy. However, this action lacks a proper instrument to ensure weight gain during pregnancy. The use of instruments for measuring would allow the possibility to follow the weight gain predicted during pregnancy with greater accuracy. One study, which compared the methods for monitoring weight gain of pregnant adolescents, found that the curve for evaluating the nutritional state of children and adolescents from the Body Mass Index, part of the World Health Organization, is an instrument that is both effective and easy to apply ${ }^{(19)}$.

The "Environment for health" axis has the objective of helping the mother to identify, comprehend, and use relational resources to adjust home environment to her needs as pregnant. In the program, the actions focused on the suitability of the home environment for the baby's arrival, in the search of residences that were safer, less overcrowded and with no exposure to tobacco, alcohol or psychological pressures. Additionally, frequently mentioned were the search for social assistance resources in situations of unemployment and the shortage of food. Notably, YMCP used the theory of bioecology as a theoretical reference in the construction of its care, which emphasizes the influence of social, community, and family context on the mother and her decisions, behaviors, and forms of caring for her children (20).

"Development of Parenting", another premise of YMCP, is also visible in a variety of actions since the beginning and over the pregnancy. Developing positive parenting is one of the most important objectives in home visit programs ${ }^{(3)}$. Young mothers for the first time that are especially socioeconomically vulnerable are more prone to drop out of visitation programs before their end, however, involving other 
members of the family in the visit and addressing issues related to parenting both minimize the chances of dropping out ${ }^{(21)}$.

Despite the low quantity of actions related to engaging parents in the study, recent initiatives demonstrate the necessity of involving both parents in the home visit and in the course of pregnancy. A review of strategies adopted to improve the engagement father in visitation programs pointed out those mobilizing their needs or expectations a schedule that is more flexible to conduct the visit and establish a relationship of trust between the visitor and the father. The study also highlighted a series of benefits that occur when both parents are involved in the program's activities, such as: better knowledge of child development, personal growth in areas such as anger control and the role of a father, capabilities to manage their relationship with the mother, and connections with opportunities for employment as well as references to other programs that can provide assistance to the Family. ${ }^{(22)}$ There is much evidence that is fundamental to include the father during pregnancy to create the bond for responsive care. ${ }^{(23)}$

The mapping of the "Family and Social Network" was an action developed during pregnancy, which allowed for the reference and elaboration of social resources available to help the pregnant woman and her family. Through the construction of a family tree and an ecomap, one can visualize the family and social network of the pregnant woman that can be utilized when the adolescent finds it to be necessary (24). Additionally, frequently mentioned were the search for social assistance resources in situations of unemployment and the shortage of food. Notably, YMP used the theory of bioecology as a theoretical reference in the construction of its care, which emphasizes the influence of social, community, and family context on the mother and her decisions, behaviors, and forms of caring for her children (20)

The premise of "Life Project" also approached during the HVs, in order to help the young pregnant to identify goals that would be relevant to her life, by encouraging the continuity of education, the job search, and family planning. Considering the importance of addressing this theme, and given that pregnancy in the teenage years, sometimes is a desire that is involved with many other lack of opportunities that could lead to serious bio-psychological complications for both the mother and child, which then makes them more prone to compromising their optimal development ${ }^{(25)}$.

While the adolescents assisted by YMCP represent those who tend to have: high social vulnerability, a lack of financial resources, a precarious family situation, insecure housing, and violence exposure, the themes proposed as premises of the program were successfully addressed during pregnancy. This is in contrast to what occurred in a home-visitation program in Australia, where the challenges of participants with a similar profile influenced the fidelity of the program. In the Australian case, the greater the number of adverse situations, the more compromised was the fidelity of the program to addressing the planned topics. ${ }^{(26)}$

Another challenge of visitation program is the transformation of family needs into practical and effective actions. The nurse visitor identifies a necessity and plans a course of action; however, articulating this action with reference to the array of services available can be a challenge in terms of making concrete 
actions. These specifics highlight a fragility at intersectoral level that is crucial to programs that aim to address the promotion of early childhood development. The evaluation of a visitation program in the United States revealed that the visitor is able to solve the family's immediate needs, such as a shortage of food or exposure to violence, even if this comes at the cost of not following the program's curriculum. However, the study also describes difficulties related to integrating the program with available services within the community, so that the families could be promptly attended. ${ }^{(27)}$

Two themes equally relevant that emerged from the study were praise and availability to address concerns. These topics relate to the bond and relationship established between the visitor and the participant. When there is a relationship of trust between the young mother and the visitor, beyond just bringing about improved results in infantile development, there are also positive impacts on family functioning and parenthood quality ${ }^{(10)}$. A relationship that is well structured with the visitor, so that they are a trusted person in the woman's life, allows for the visitor to help resolve conflicts and encourage the activation of the woman's social network when necessary. ${ }^{(26)}$

It is clear from the reports prepared by the nurses, that the planning of actions was based on interaction and dialogue, in an attempt to apply pre-existing knowledge and reflect on previous experiences. Where there is a learning process, a Freirean perspective, involves motivational and emotional-relational aspects, as well as relationships established centered on an action. In this sense, learning is a reflective process, which involves many different actors and perspectives, in order to produce critical knowledge and meaning, a process that matches the perspective of family-centered care. ${ }^{(28)}$

\section{Conclusion}

Overall, the study highlights that the themes discussed in the HVs match those proposed by the YMCP. The study confirmed that the majority of actions planned by the visitors in fact realized in the following visit. This is the most important indicator of fidelity of the actions offered.

The interaction established between the adolescent mother and the visiting nurse is fundamental for care. In this study, the "praise" which appeared as a recurring action undertaken by the nurse could serve as a "facilitator" of the bond and interaction between the adolescent mother and the visiting nurse.

The focus on the premise related to caution with health was extremely evident during the period analyzed (prenatal), and this can be explained by the intense physical changes that the adolescent experiences at this stage, which seem to be "the most important" part of this period. When considering questions like, "how can I care for the baby; who is going to help me when I need to go back to study; should I go back to school or not" among others, these seem to be secondary issues during this phase.

The innovativeness of this study is presenting the potential and limits of structured interventions in a longitudinal context of home-based care. The central question was to verify how the uncertainties of daily domestic life, and its various demands, would interfere with the delivery of the actions planned as part of 
the YMCP and to what degree the visiting nurse would be able to stay faithful to the premises of the program.

The limitations of the study include that only the protocols during the pregnancy period investigated, even though the duration of the intervention is up to two years of the child's life.

The impact of nursing in this program stems from the theoretical and practical capabilities of the nurse to promote parenting. Thus, this prompts consideration of graduate and post-graduate courses in order to reflect the training they provide in terms of teaching and research.

For the next study, we suggest that the databases are structured and the systems for maintaining records allow for the possibility of evaluating and measuring actions over a longer period.

\section{Conclusions For Practice}

The protocols for the HVs should be flexible when considering the needs of the families and individuals, especially those that are in a position of high social and economic vulnerability. Beyond this, the HVs should adopt strategies in terms of their approach and intervention that adapt to the reality of the necessities of the families living in certain realities, whether these are social, psychological, or healthrelative. This helps guarantee that the intervention has positive effects for the individual, family, and society. ${ }^{(13)}$

\section{Abbreviations}

HV

Home Visit

YMCP

Young Mother Caregivers Program

\section{Declarations}

\section{ETHICS APPROVAL AND CONSENT TO PARTICIPATE}

This research follows the guidelines set out by Resolution 510/2016, and approved by the Committee of Ethics in Researcher of the School of Medicine of São Paulo University (Brazil), registered under the number 41573015.0.0000.0065 and the advice number 1.397.051. All participants signed an informed consent form agreeing to participate in the study.

\section{CONSENT FOR PUBLICATION}

Not applicable. 
AVAILABILITY OF DATA AND MATERIAL

The datasets used and/or analyzed during the current study are available from the corresponding author on request.

\section{COMPETING INTERESTS}

The authors declare that they have no competing interests.

FUNDING

The YMCP program was funded by the National Institute of Developmental Psychiatry for Children and Adolescents at the University of São Paulo School of Medicine in Brazil. Funding agencies had no role in this study.

\section{AUTHORS' CONTRIBUTIONS}

LDS was primarily responsible for data analysis and published work. LAF, LAS and AMC designed the study, interpreted the data. SV was responsible for critically review and transcription. ECM and FSPA critically reviewed the report and all authors approved the final manuscript as presented.

\section{ACKNOWLEDGEMENTS}

The authors give their special thanks to all researchers and program coordinator that supported them in all stages of the study.

\section{References}

1. Kristof N. The way to beat the poverty. 2014. The New York Times. http://www.nytimes.com/2014/09/14/opinion/sunday/nicholas-kristof-the-way-to-beat-poverty.html. Accessed 10 sep 2016.

2. Britto PR, Lye SJ, Proulx K, Yousafzai AK, Matthews SG, Vaivada T. Nuturing Care: promoting early childhood development. The Lancet. 2017. doi:10.1016/S0140-6736(16)31390-3.

3. Sama-Miller E, Akers L, Mraz-Esposito A, Zukiewicz M, Avellar S, Paulsell D, Del Grosso P. Home Visiting Evidence of Effectiveness Review: Executive Summary. U.S. Department of Health and Human Services. 2017. https://www.acf.hhs.gov/opre/resource-library/search?sort=recent. Accessed jun 2018.

4. Britto P, Singh M, Dua T, Kaur R, Yousafzai AK. What implementation evidence matters: scalling-up nurturing interventions that promote early childhood development. Ann N Y Acad Sci. 2018. 
doi:10.1111/nyas.13720.

5. Richter L, Daelmans B, Lombardi J, Heymann J, Boo F, Behrman J, Lu C, Lucas J, Perez-Escamilla R, Dua T, Bhutta Z, Stenberg K, Gertler P, Darmstadt G. Investing in the foundation of sustainable development: pathways to scale up for early childhood development. The Lancet. 2017. doi:10.1016/S0140-6736(16)31698-1.

6. Duffee JH, Mendelsohn AL, Kuo AA, Legano LA, Earls MF. Early Childhood Home Visiting Pediatrics. 2017. doi:10.1542/peds.2017-2150.

7. Maria Cecília Souto Vidigal Foundation. Science Center for Childhood. Home visit as a strategy to promote development and parenting in early childhood. Sao Paulo: FMCSV; 2018.

8. Kebian L, Acioli S. Home visits by Family Health Strategy nurses and community health agents. Rev Eletr Enferm. 2014. doi:https://doi.org/10.5216/ree.v16i1.20260.

9. Schaffer MA, Goodhue A, Stennes K, Lanigan C. Evaluation of a public health nurse visiting program for pregnant and parenting teens. Public Health Nurs. 2012. doi:10.1111/j.1525-1446.2011.01005.x.

10. Zapart S, Knight J, Kemp L. 'It Was Easier Because I Had Help': Mothers' Reflections on the LongTerm Impact of Sustained Nurse Home Visiting. Maternal Child Health Journal. 2015. doi:10.1007/s10995-015-1819-6.

11. Saïas T, Lerner E, Greacen T, Simon-Vernier E, Emer A. Evaluating Fidelity in Home-Visiting Programs a Qualitative Analysis of 1058 Home Visit Case Notes from 105 Families. Plos One. 2012. doi:https://doi.org/10.1371/journal.pone.0036915.

12. Azzi-Lessing L. Home visitation programs: Critical Issues and Future Directions. Early Child Research Quarterly. 2011. doi:https://doi.org/10.1016/j.ecresq.2011.03.005.

13. Drummond JE, Weir AE, Kysela GM. Home Visitation Practice: Models, Documentation, and Evaluation. Public Health Nurs. 2002. doi:10.1046/j.1525-1446.2002.19004.x.

14. Korfmacher J, Laszewski A, Sparr M, Hammel J. Assessing Home Visit Program Quality. Final Report to the Pew Center on the States. https://www.researchconnections.org/childcare/resources/26454. Acessed 2018 may 07.

15. Moraes R. Content Analysis. Revista Educação. 1999;22:37; 7-32.

16. Henderson S, Segal EH. Visualizing qualitative data in evaluation research. New Dir Eval. 2013. doi:10.1002/ev.20067.

17. Fracolli LA, Reticena KO, Abreu FCP, Chiesa AM. The implementation of a home visits program focused on parenting: an experience report. Rev Esc Enferm USP. 2018. doi:http://dx.doi.org/10.1590/S1980-220X2017044003361.

18. Pinheiro DGM, Pola TBC, Silva CFA, Silva LA, Chiesa AM, Fracolli LA. Content validation of home visiting program for mother-child dyad. Cogitare Enferm. 2018. doi:http://dx.doi.org/10.5380/ce.v23i2.54055.

19. Pinho-Pompeu M, Paulino D, Morais S, Crubelatti M, Pinto e Silva J, Surita F. How to classify BMI among pregnant adolescents? A prospective cohort. Public Health Nutrition. 2019. 
doi:10.1017/S136898001800276821.

20. Bronfenbrenner U, Morris PA. The ecology of developmental processes. In: Damon W, Lerner RM, editors. Handbook of child psychology: theoretical models of human development. 6th ed. New Jersey: John Wiley \& Sons; 2006. pp. 993-1028.

21. Brand T, Jungmann T. Implementation differences of two staffing models in the german home visiting program "pro kind". J Community Psychol. 2012. doi:10.1002/jcop.21489.

22. Heather S, Gearing M, Peters HE, Heller C, Healy O, Pratt E. Approaches to Father Engagement and Fathers' Experiences in Home Visiting Programs. OPRE Report. 2015.

https://www.acf.hhs.gov/sites/default/files/opre/20151130_fahv_report_finalized_b508.pdf Acessed 2018 dec 10.

23. Barlow J, Schrader McMillan A, Kirkpatrick S, Ghate D, Barnes J, Smith M. Health-led interventions in the early years to enhance infant and maternal mental health: a review of reviews. Child Adolesc Mental Health. 2010. doi:https://doi.org/10.1111/j.1475-3588.2010.00570.x.

24. Nascimento LC, Dantas IRO, Andrade RD, Mello DF. Genogram and ecomap: brazilian nursing contributions. Texto Contexto Enfermagem. 2014. doi:https://dx.doi.org/10.1590/S010407072014000100025.

25. Azevedo WF, Diniz MB, Fonseca ESVB, Azevedo LMR, Evangelista CB. Complications in adolescent pregnancy: systematic review of the literature. Einstein. 2015. doi:https://dx.doi.org/10.1590/S167945082015 RW3127.

26. Zarnowiecki D, Nguyen H, Catherine H, Boffa J, Segal L. The Australian Nurse-Family Partnership Program for aboriginal mothers and babies: Describing client complexity and implications for program delivery. Midwifery. 2018. doi:https://doi.org/10.1016/j.midw.2018.06.019.

27. Bryant K, Chung G, Lanier P, Verbiest S. North Carolina Early Home Visiting Landscape Analysis: Strengthening Systems to Support Families. Jordan Institute for Families. 2018. http://jordaninstituteforfamilies.org/wp-content/uploads/2018/09/NC-HV-Study-09_07_18FINAL.pdf Acessed 2019 fev 20.

28. Cunha RCOB, Pezzato LM. Educational practices and meaning production. In: Pelicioni MCF, Mialhe FL (org.). Health Education and Promotion: theory and practice. Sao Paulo: Santos; 2012. pp. 26586.

\section{Figures}




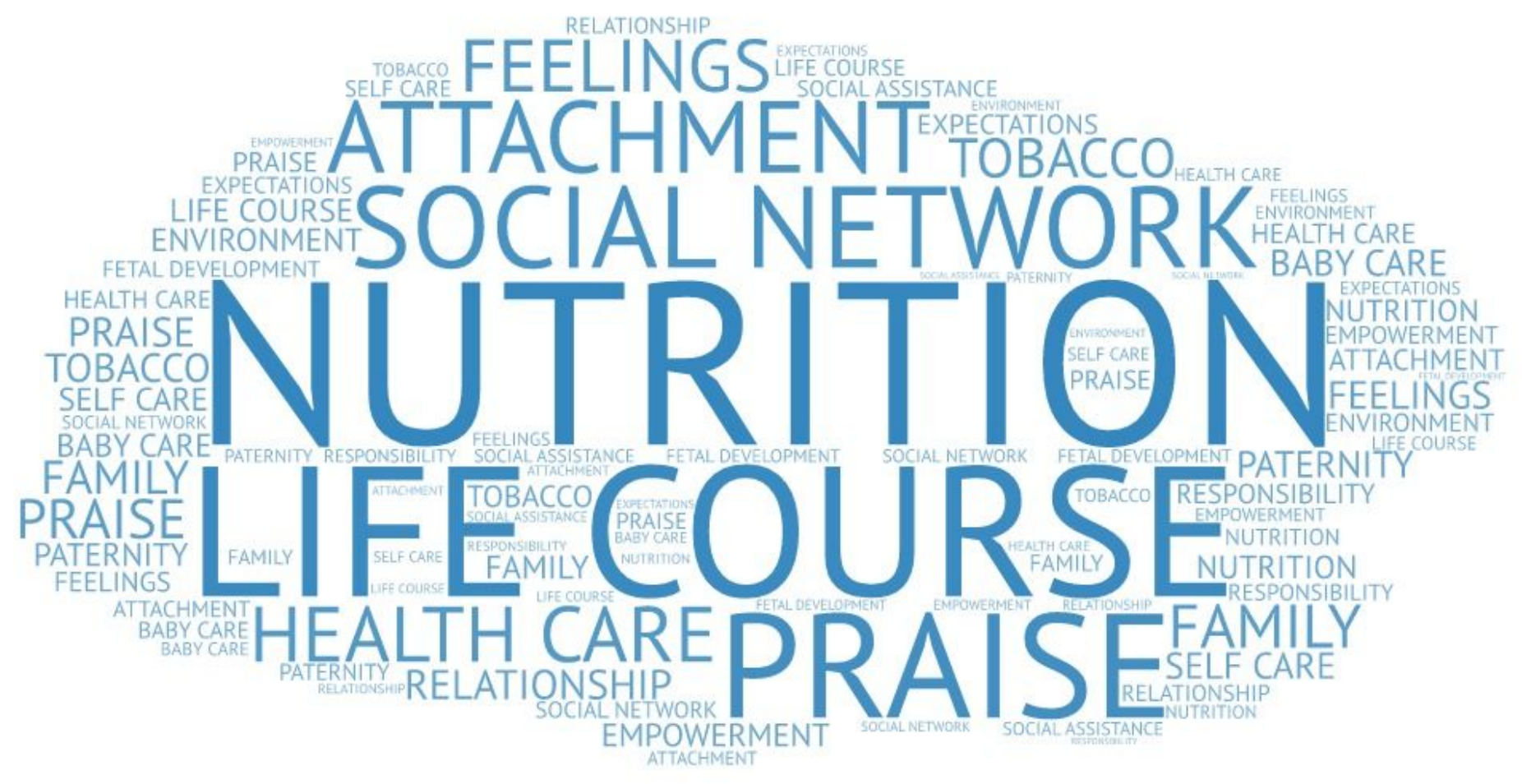

Figure 1

Themes from home visits during the first gestational trimester. Sao Paulo, 2020.

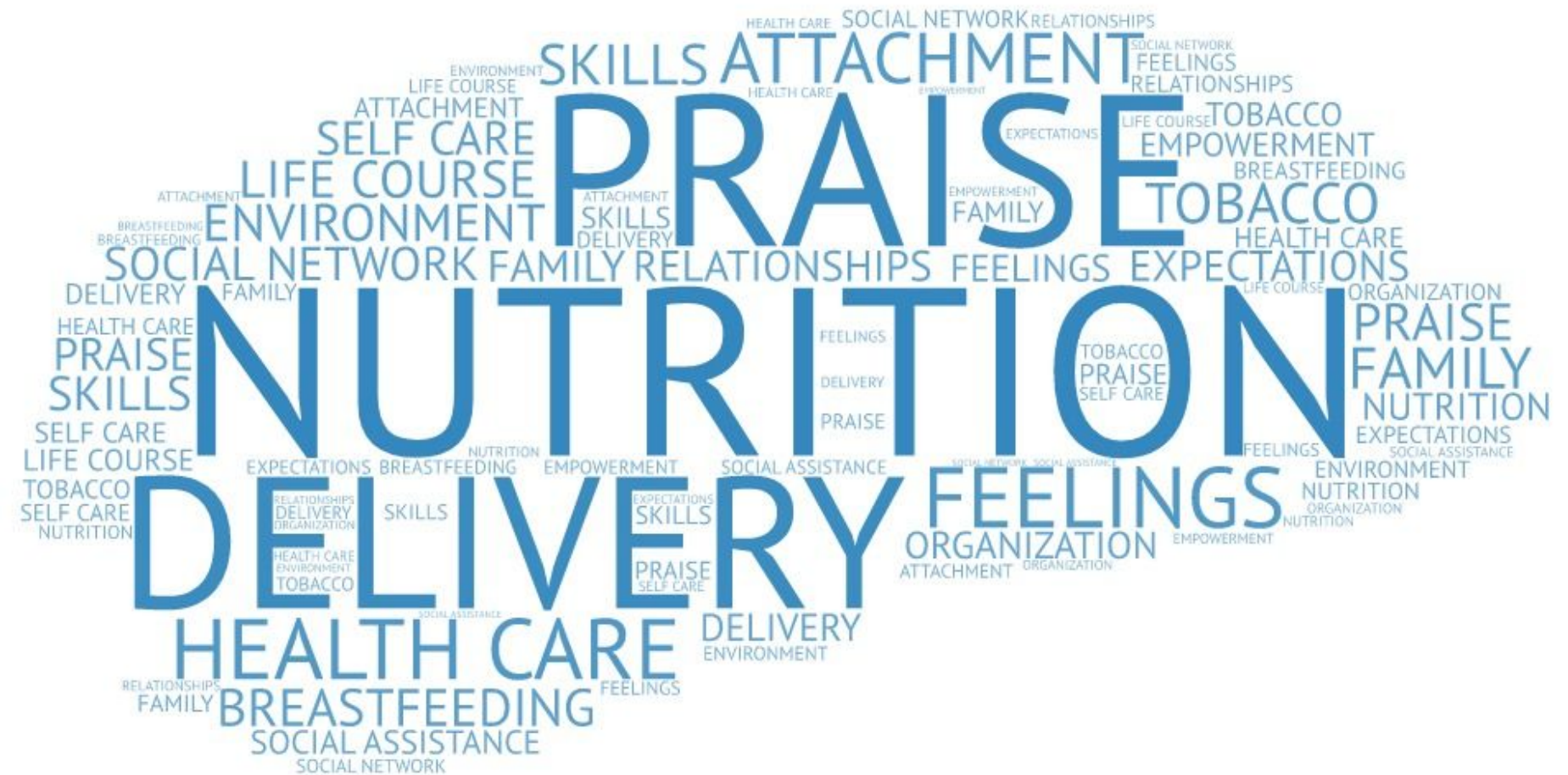

Figure 2

Themes from home visits during the second gestational trimester. Sao Paulo, 2020. 


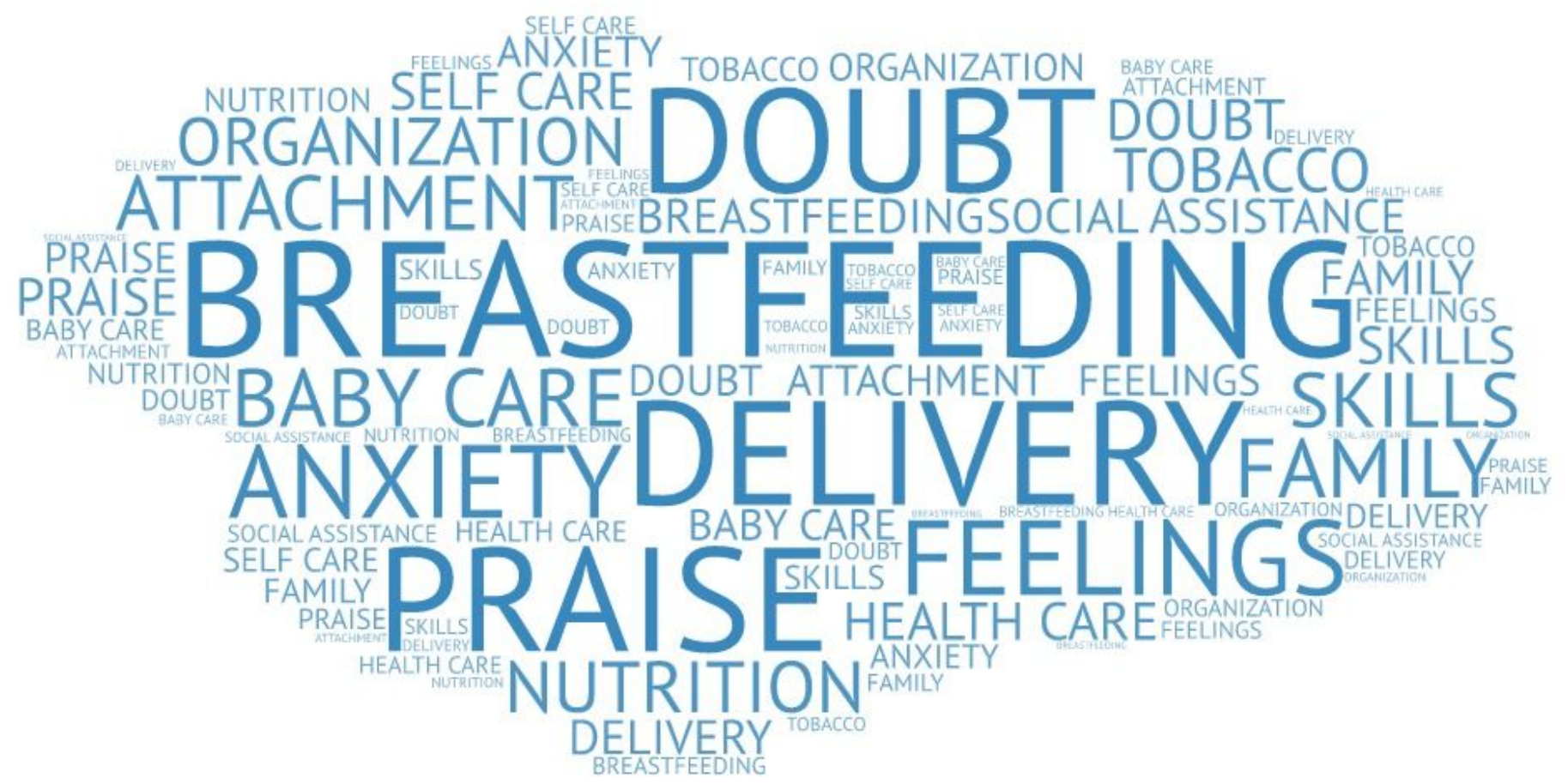

Figure 3

Themes from home visits in the third gestational trimester. Sao Paulo, 2020. 